\title{
An Integrated Study Employing Histopathological, Immunohistocytochemical and Radioimmunoassay Analyses of Atrial Natriuretic Peptide in the Right and Left Atria in Patients with Mitral Valve Disease
}

\author{
Makoto Nagata, M.D., Michiaki Hiroe, M.D., Mitsuhide Naruse, M.D. \\ Zu-Xi Yu, M.D., Morie Sekiguchi, M.D., Koshichiro Hirosawa, M.D. \\ Hitoshi Koyanagi, M.D., Akimasa Hashimoto, M.D., Masahiro Endo, M.D. \\ and Kazuo Shizume, M.D.
}

\begin{abstract}
To clarify the production mechanism of atrial natriuretic peptide (ANP) in right (RA) and left atria (LA) in mitral valve disease, histopathological and immunohistocytochemical analyses were performed and ANP levels were investigated by radioimmunoassay (RIA) in 28 patients. Atrial tissues were obtained during mitral valve replacement. ANP-like immunoreactivity of the myocytes applied by the avidin-biotin peroxidase complex method was observed around the nuclei of the atrial myocytes. Electronmicroscopically, immunoreactivity was observed in atrial specific granules. Light-microscopically determined intensity of the immunoreactivity was classified into 4 grades and the intensity in 100 myocytes was expressed by adding the scores of each myocyte. Mean right atrial pressure was positively correlated with the activity score in RA $(r=0.80)$. Pulmonary capillary wedge pressure was not correlated with the score in LA. The score in RA was significantly higher than that in LA. The ANP level in RA investigated by RIA was also higher than that in LA. Histopathological findings such as myocyte hypertrophy, degeneration and interstitial fibrosis were more severe in LA than in RA. In conclusion, longstanding atrial overloading, especially in LA, caused severe pathological damage, resulting in a smaller production of ANP. Much more ANP may be produced from RA in long-standing mitral valve disease.
\end{abstract}

$\mathbf{A}^{\mathrm{s}}$ all aspects of the relationship between atrial natriuretic peptide (ANP) and heart diseases have not been clarified, we examined right and left atrial tissues from patients with mitral valve disease, employing the avidin-biotin peroxidase complex $(\mathrm{ABC})$ method on the post-

Key words:

Atrial natriuretic peptide

Mitral valve disease

Immunohistocy tochemistry

Radioimmunoassay

Right and left atria embedded sections. Details of this method were previously reported! In the present study, we also analysed atrial levels of ANP by radioimmunoassay (RIA).

\section{MATERIALS AND METHOD}

Atrial tissues were excised at cardiac surgery from 17 patients with mitral stenosis (MS) and from 11 with mitral regurgitation (MR). Specimens were obtained from the right auricular

The Heart Institute of Japan, Department of Radiology and Endocrinology, Tokyo Women's Medical College, Tokyo, Japan

Grant: This work was supported by a research grant from the Japan Promotion Society for Cardiovascular Diseases.

Mailing address: Makoto Nagata, M.D., The Heart Institute of Japan, Tokyo Women's Medical College, 8-1 Kawada-cho, Shinjuku-ku, Tokyo 162, Japan 

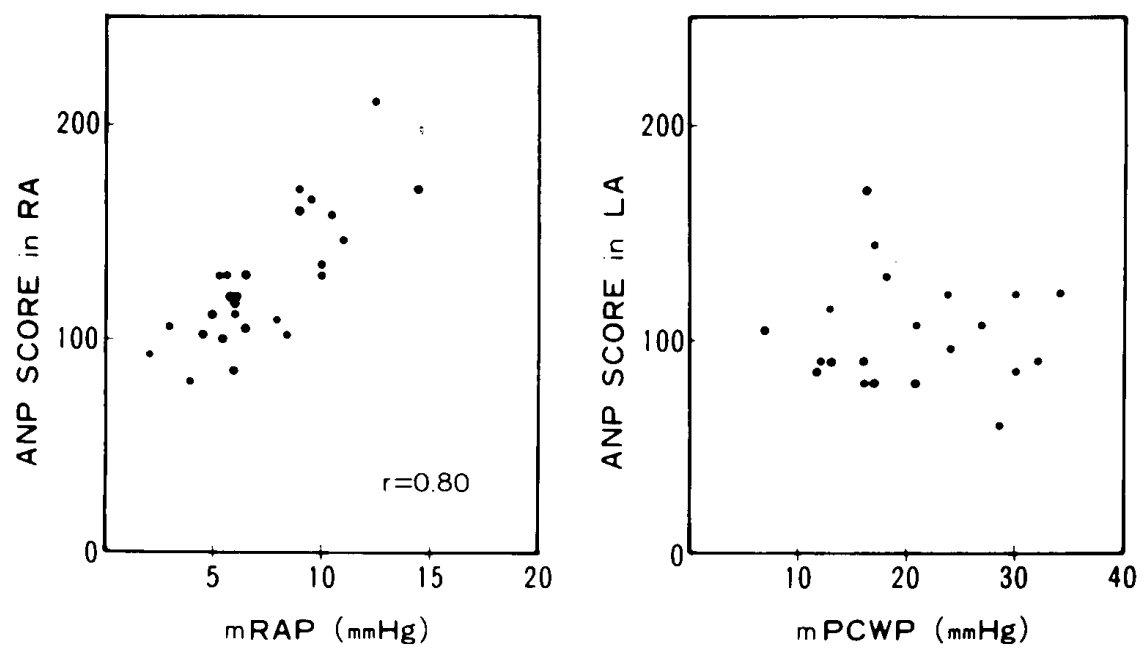

Fig.1 A: Relationship between mean right atrial pressure (mRAP) and lightmicroscopically determined immunoreactivity score of atrial natriuretic peptide (ANP SCORE) in right atrium (RA) $(n=26)$.

B: Relationship between mean pulmonary capillary wedge pressure (mPCWP) and ANP SCORE in left atrium (LA) $(n=21)$.

tissue when the tube for extracorporeal circulation was inserted. Left atrial tissue was also taken during left atriotomy. The specimens were divided into three parts. One, for histopathologic and immunohistochemical studies, was fixed in $10 \%$ buffered formalin. Another, for electronmicroscopical and immunocytochemical investigations, was pre-fixed in $3 \%$ glutaraldehyde and post-fixed with $1 \%$ OsO4. These specimens were processed for the $\mathrm{ABC}$ method using antiserum IgG against $\alpha$-human ANP as used postembedding method! The third part of each specimen was used for measurement of atrial levels of ANP by RIA?

The stained specimens for light microscopy were evaluated for the intensity of the immunoreactivity and graded as follows; 0: absent, 1+: slight, ++: moderate, +++: high. Scoring was done by adding the results from 100 cardiac myocytes. Intra- and inter-observer variance of the quantitative assessment for the score was evaluated and no statistical difference was found.

Results of the histochemical analysis were compared with those of the histopathological and ultrastructural observations, and clinical data, such as NYHA functional class, ECG, chest X-ray, echocardiography and hemodynamic parameters, measured by right and left heart catheterization, a few weeks before the operation. For quantification of the histopathological findings, the breadth of the atrial muscle cells was evaluated by measuring the diameter across the nucleus, and the percent fibrosis was determined by observing 1000 points through the microscope at 200 magnification, using the point-counting method. The severity of degeneration of the cardiac myocytes was evaluated by our own method?

All values were expressed as mean $\pm 1 \mathrm{SD}$. Statistical analysis was performed by the standard method using the paired t-test and the linear regression analysis.

\section{RESULTS}

In both right and left atrial muscle, small particles were found around the nuclei showing immunoreactivity of ANP. No immunoreactivity was found in the right ventricle. Ultrastructurally, atrial specific granules (AG) were distributed around the nucleus and some were scattered in between the myofibrils and in the subsarcolemma. The ANP immunoreactivity was found to be identical to the AG distribution!

The relationship of the lightmicroscopically determined immunoreactivity of the ANP and clinical data was analyzed. The scores of the ANP activity in the right atrium showed a close correlation with the right atrial mean pressure with a relation coefficient of 0.80 (Fig. 1A). The intensity in the patients with atrial fibrillation and tricuspid regurgitation was much higher than in the other patients. However, pulmonary capillary wedge pressure did not correlate with the ANP scores in the left atrium (Fig. 1B). Right atrial intensity was significantly 


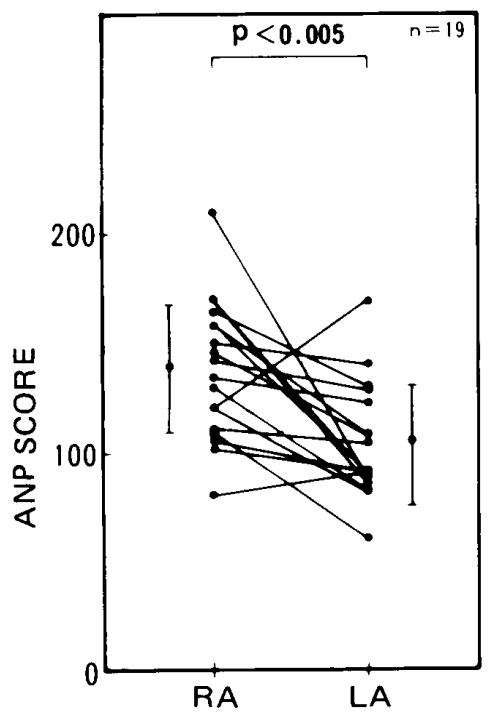

Fig. 2 Comparison of ANP SCORE (see Fig. 1) in right atrium (RA) and left atrium (LA).

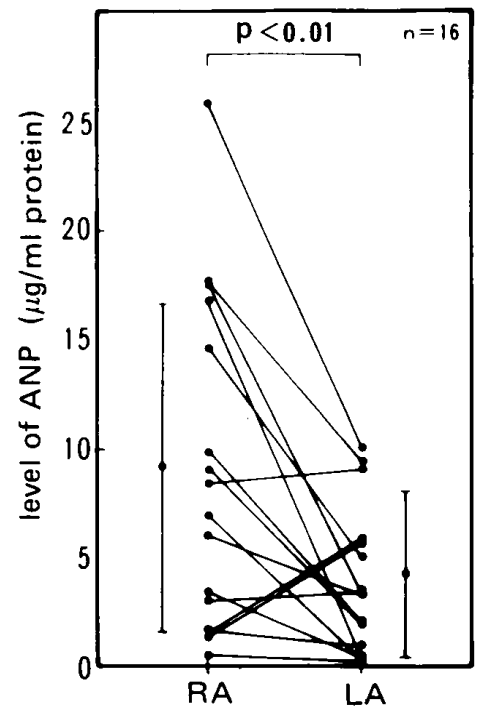

Fig. 3 Comparison of atrial levels of ANP analyzed by radioimmunoassay in right atrium (RA) and left atrium (LA).

TABLE I CLINICAL DATA OF THE PATIENTS

\begin{tabular}{llcc}
\hline \hline & & $M S(n=17)$ & $M R(n=11)$ \\
\hline Age & & $51 \pm 10$ & $50 \pm 10$ \\
NYHA & $(I: I I: I I I: I V)$ & $0: 10: 6: 1$ & $1: 7: 2: 1$ \\
ECG & $(S R:$ af $)$ & $2: 15$ & $2: 9$ \\
CTR on $X$-ray & $(\%)$ & $64 \pm 12$ & $64 \pm 7$ \\
LAD on $U C G$ & $(m m)$ & $53 \pm 17$ & $58 \pm 5$ \\
$m R A P$ & $(m m H g)$ & $7.7 \pm 3.1$ & $7.0 \pm 2.8$ \\
$m P C W P$ & $(m m H g)$ & $23.9 \pm 6.6$ & $14.5 \pm 4.5$ \\
TR & & 8 & 2
\end{tabular}

$M S=$ mitral stenosis $; \quad M R=$ mitral regurgitation; $N Y H A=$ New York Heart Association functional class; $E C G=$ electrocardiogram; $S R=$ sinus rhythm; af = atrial fibrillation; $C T R$ on $X$-ray = cardiothoracic ratio; LAD on UCG = left atrial dimention assessed by echocardiography using either the $M$ mode section or the two-dimentional estimate; $m R A P=$ mean right atrial pressure evaluated by right heart catheterization; $m P C W P=$ mean pulmonary capillary wedge pressure evaluated by right heart catheterization; $T R=$ tricuspid regurgitation.

higher than left atrial intensity (Fig. 2).

The radioimmunoassay revealed that ANP levels in right atrial tissue $(9.13 \pm 7.54 \mu \mathrm{g} / \mathrm{mg}$ protein) was significantly higher than in the left atria $(3.8 \pm 3.4 \mu \mathrm{g} / \mathrm{mg}$ protein $)(\mathrm{p}<0.01)$ (Fig. 3).

In addition, histopathological analysis revealed that the diameter of myocytes in right and left atria was $14.0 \pm 1.7 \mu \mathrm{m}, 21.0 \pm 5.3 \mu \mathrm{m}$, respectively ( $\mathrm{p}<0.001)$, and percent fibrosis was $28.9 \pm 7.9 \%, 42.8 \pm 9.4 \%$, respectively $(\mathrm{p}<0.01)$. Degeneration of myocytes was much more pronounced in left than in right atrial myocardium.

\section{DISCUSSION}

Morphological demonstration of ANP was carried out in human atrial tissue by Cantin et al using immunohistocytochemical method. ${ }^{4}$ However, this tissue processing method was complex. Our application of the $\mathrm{ABC}$ method using tissue blocks for routine histopathological examination has simplified the tissue processing. With this method, we could demonstrate the ANP activity by both light and electronmicroscopical methods. There is no report of investigations of the ANP activity in both right and left atrial tissues in the 
same case. We found that the activity was present in both atria. Furthermore, our present study revealed that the ANP activity was higher in the right than in the left atrium, in mitral valve disease. Similar results were obtained in our RIA analysis. It should be mentioned here that in all patients, the left atrium was severely demaged because of the presence of long-standing disease. Our semiquantitative evaluation revealed a high correlation between immunoreactivity and right atrial pressure, but no close correlation with the left atrial pressure. The low immunoreactivity of the left atrial ANP is due to longstanding pressure and volume overloading in mitral valve disease leading to the decompensated stage of hypertrophy and degeneration of the myocytes. It is, therefore, speculated that the increase in the ANP activity in the right atrium indicates overloading to the right atrium which is causing compensatory hypertrophy and hyperfunction of the right atrial myocytes?

\section{Acknowledgement}

The English correction of this manuscript by Miss
Barbara Levene is appreciated.

\section{REFERENCES}

1. NAGATA M, HIROE M, YU ZX, SEKIGUCHI M, HIROSAWA K: Immunohistochemical localization of alpha-human natriuretic peptide in human atrium obtained from endomyocardial biopsy and cardiac surgery. Heart and Vessels (40 (1) 1988, in press)

2. NAKAO K, SUGAWARA A, MORII N, SAKAMOTO M, SUDA M, SONEDA J, BAN T, KIHARA M, YAMORI Y, SHIMOKURA M, KISO Y, IMURA $\mathrm{H}$ : Radioimmunoassay for $\alpha$-human and rat atrial natriuretic polypeptide. $B B R C$ 124: 815,1984

3. SEKIGUCHI M, HIROE M, MORIMOTO S: On the standardization of histopathological diagnosis and semiquantitative assessment of the endomyocardium obtained by endomyocardial biopsy. Bulletin of the Heart Institute Japan 55, 1979-80

4. CANTIN M, GUTOWSKA J, MILES RW, LEDOUX S, MINLI S, CHAPEAU C, GARCIA R, HAMET $P$, GENEST J: Immunocytochemical localization of atrial natriuretic factor in the heart and salivary glands. Histochemistry 80: 113, 1984

5. SEKIGUCHI $M$, NISHINO $H$, NISHIKAWA $T$, MORIMOTO S, HIROE M: Age-associated myocardial changes in various heart diseases. Jpn Circ J 50: 1032, 1986 